\title{
GESTURE CONTROLLED DRAWER FOR SMART HOME
}

\author{
Sudipta Sahana ${ }^{1}$, Suvam Basak ${ }^{2}$, Sreeja Chowdhury ${ }^{3}$
}

\begin{abstract}
Hand gesture recognition systems were developed with applications in fields of sign language recognition, driver assistance, smart environments, video games, medical instrumentation, and other humancomputer interfaces. Hand gesture recognition systems commonly use depth information for background removal purposes using a Finger-Earth Mover's Distance (FEMD) for recognizing static poses. In this paper we have proposed a smart drawer that makes home smarter. The main idea works in line with gesture recognition technique. User choice based additional security enhancement technique has been implemented by face recognition. Two different gestures are preinstalled for opening and closing the drawer. After matching the gesture, if security is enabled face recognition is required for opening the drawer. A small camera is installed at a particular place on the drawer in capturing mode. The camera gets continuous footage of actions near the drawer and as it has been designed for gesture recognition already so it try to match the capture gesture with the preinstall gesture and do accordingly.
\end{abstract}

Keywords - Hand gesture recognition systems, Finger-Earth Mover's Distance, Smart Drawer, Security, Camera

\section{INTRODUCTION}

This is the $21^{\text {st }}$ century we are residing in and this era specializes in evolution in the technological sphere. In whichever direction we look, electronic means replacing the traditional apparatus we are using. Technological advancement has given us different ways to view things in a simpler manner. Keeping this in mind we have thought of areas where technology could be put in better use so that it helps us resolve time and space issues. Involvement of technology in regular usable home goods could help us in various ways.Researches show that gesture based applications can be used for many different things, entertainment, controlling home appliance, tele-care, tele-health, elderly or disable care. The scope of the application shows us the importance of more researches in a gesture controlled system. Most applications are to replace traditional input devices likekeyboard and mouse, accessible application for elderly-disable like accelerometer. Initial applications were on pc applications for text edit presentation. Gesture visualization has been included for feedback and training. Using digital camera rather than sensor has provided new dimension to develop gesture based user interface development. Now people can interact with any media using gesture to control wide range of applications. We have got gesture based commercial products in 2003. People get gesture based hardware and software with choice. So here we present an idea that will make a normal drawer more useful, secured and easy to access. Smart technology involves access to the drawer using machine learning. This drawer will only open for the particular person who has been granted access to it and that too without the need to put in mechanical effort but just using simple gestures to open and close it. Thus we can call this, a smart drawer.

The language used supports multiple programming paradigms, including object-oriented, imperative and functional programming or procedural styles. If features a dynamic type system and automatic memory management and has a large and comprehensive standard library.

A library of many inbuilt functions mainly aimed at real time image processing is used.Hand gesture recognition systems were developed with applications in fields of sign language recognition, driver assistance, smart

${ }^{1}$ Department of Computer Science and Engineering JIS College of Engineering, Kalyani, West Bengal, India

${ }^{2}$ Department of Computer Science and Engineering JIS College of Engineering, Kalyani, West Bengal, India

${ }^{3}$ Department of Computer Science and Engineering JIS College of Engineering, Kalyani, West Bengal, India 
environments, video games, medical instrumentation, and other human-computer interfaces. Hand gesture recognition systems commonly use depth information for background removal purposes. proposed using a FingerEarth Mover's Distance (FEMD)for recognizing static poses. Hand detection is commonly performed using skin analysis, depth information is used to segment the hand and estimate its orientation using PCA with a refinement step. The classification of static gestures is performed using an average neighbourhood margin maximization classifier combined with depth and hand rotation cues, a nearest neighbour classifier with a dynamic time warping (DTW) measure was used to classify dynamic hand gestures of digits from zero to nine. A Hidden Markov Model(HMM) may also be used for gesture modelling. Features of global image statistics or grid coverage, and a randomized decision forest for depth-based static hand pose recognition. There has been some work in adapting colour descriptors to be more effective when applied to depth data.

The rest of the paper is organized as follows. Section II contains the literature survey. Proposed work on gesture recognition has been described in section III. Experimental results are presented in section IV. Concluding remarks are given in section $\mathrm{V}$.

\section{RELATED WORKS}

The ways of recognizing the gesture can be considered as a significant progress of the technology. Progress of image processing technology has played an important role here. Gestures have been captured by using infrared beams, data glove, still camera, wired and many inter-connected technologies like gloves, pendant, infrared signal network server etc. in the past. Recent vision technique, video and web cam based gesture recognition has made it possible to capture any intuitive gesture for any ubiquitous devices from the natural environment with 3D visualization. Researchers have been done on hand recognition and gesture recognition in India as well as outside India.

A recent research describes the primary and secondary driving task together with Human Machine Interface (HMI) trends and issues which are driving automotive user interface designers to consider hand gesture recognition as a realistic alternative for user controls. A number of hand gesture recognition technologies and applications for Human Vehicle Interaction (HVI) are also discussed including a summary of current automotive hand gesture recognition research.[1]

Also, trajectory-based interactions, such as navigating through nested-menus, drawing curves, and moving in $3 \mathrm{D}$ worlds, are becoming common tasks in modern computer interfaces. Users performances in these tasks cannot be successfully modeled with Fits law as it has been applied to pointing tasks. Therefore we explore the possible existence of robust regularities in trajectory-based tasks.[2]

Gesture based interfaces promise to increase the efficiency of user input, particularly in mobile computing where standard input devices such as the mouse and keyboard are impractical. This paper describes an investigation into the low-level physical properties of linear 'flick' gestures that users create using mouse and pen input devices. The study was motivated by our need to determine sensible constraints on values such as the magnitude, timing, and angular accuracy of gestures[3]

The proposed scheme is presented for recognizing hand gestures using the output of a hand tracker which tracks a rectangular window bounding the hand region. A hierarchical scheme for dynamic hand gesture recognition is proposed based on state representation of the dominant feature trajectories using an a priori knowledge of the way in which each gesture is performed.[4]

A method for developing a gesture-based system using a multi-dimensional hidden Markov model (HMM). Instead of using geometric features, gestures are converted into sequential symbols. HMMs are employed to represent the gestures and their parameters are learned from the training data. Based on "the most likely performance" criterion, the gestures can be recognized through evaluating the trained HMMs.[5]

A gesture corresponds to a particular pattern of head movement. The facial plane is tracked using a parameterized model and the temporal sequence of three image rotation parameters are used to describe four gestures. A dynamic vector quantization scheme was implemented to transform the parameters into suitable input data for the HMMs. Each model was trained by the iterative Baum-Welch procedure using 28 sequences taken from 5 persons. [6]

This is an approach to 2D gesture recognition that models each gesture as a Finite State Machine (FSM) in spatialtemporal space. The model construction works in a semi-automatic way. The structure of the model is first manually decided based on the observation of the spatial topology of the data then refined iteratively between two stages: data segmentation and model training. [7] 
Gestures can be divided into postures, where the connguration of the hand is static, and true gestures, where the exion of the ngers and the hand posi-tion/direction are changing dynamically. A recognition model based on a hybrid artiicial neural network combining a radial basis function and a Bayesian clas-siier network is developed and tested with various forms of pre-processing or input representation. The results suggest that dynamic gesture recognition is feasible even for complex gestures, but that context information may be needed for reliable recognition. Gesture interaction is a very diverse eld, where many applications and methods are related more by the fact that they are said to deal with gesture interaction than any inherent similarity[8]

\section{PROPOSED WORK}

Image processing is the process of manipulating image data in order to make it suitable for computer vision applications or to make it suitable to present it to humans. . Basic steps for a typical computer vision application as follows.
2. Image acquisition
3. Image manipulation
4. Obtaining relevant information
5. Decision making

The working of the smart drawer involves two parts:

$$
\begin{aligned}
& \Rightarrow \text { The digital part } \\
& \Rightarrow \quad \text { The mechanical part }
\end{aligned}
$$

The digital part involves the face recognition and gesture recognition paradigms. It also involves how the drawer driver is linked with the opening and closing methodologies..

The mechanical part involves how the driver has been programmed and pulley system has been used to bear the particular weight of the drawer when it is put in 'on' position so it takes the load that is the drawer back inside or keeps it inside, and when it is 'off' it pushes and keeps the drawer outside.

Here we make a smart drawer by teaching it how it has to work providing it instructions and programming it.We code in a manner that gesture is recognisable.We then code in a manner that the owner's face is also recognisable This is done by a small camera located at a particular place on the drawer.The camera is always on.The camera gets continuous footage of actions near the drawer.The camera has been designed for gesture recognition already so it checks if gesture matches the open gesture it has been taught.If it matches then it checks for the face recognition with owner's face. The drawer unlocks and opens if matched.If it doesn't match drawer does not open. When drawer is open already, another gesture provided as close gesture closes the drawer.

This is how the gesture recognition works:

\section{Step 1: Capture frames and convert them to grayscale}

Our ROI is the the hand region, so we capture the images of the hand and convert them to grayscale. We convert an image from RGB to grayscale and then to binary in order to find the ROI i.e. the portion of the image we are further interested for image processing

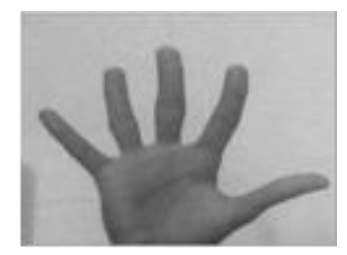

\section{Step 2: Extract ROI: Blurring and Thresholding}

By blurring, we create smooth transition from one color to another and reduce the edge content. 
We use the threshold for image segmentation, to create binary images from grayscale images.

\section{Step 3: Find contours}

A contour is the boundary following our ROI

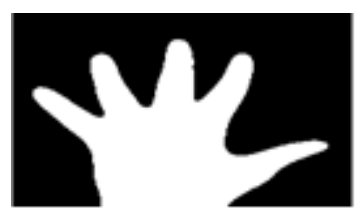

\section{Step 4: Find convex hull and convexity defects}

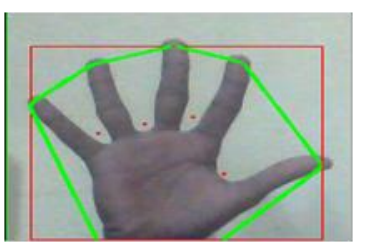

We now find the convex points and the defect points. The convex points are generally, the tip of the fingers. But there are other convex point too. So, we find convexity defects, which is the deepest point of deviation on the contour. By this we can find the number of fingers extended and then we can perform different functions according to the number of fingers extended

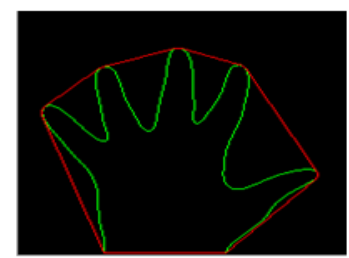

After the gesture has been recognised, machine learning has been used to enable extra secured access which operates for the face recognition.

A database is created for only the particular people we want to enable access to the drawer. The various angles of faces has been taken and saved as files to that database. Using machine learning the faces get recognised. If someone's face doesn't match he or she can't open the drawer even if the gesture has matched.

The user has right to choose whether he or she wants only gesture enabled drawer or gesture added to face checking enabled drawer.

* There are good business opportunities because these are:

$$
\begin{aligned}
& \Rightarrow \text { Smart } \\
& \Rightarrow \text { Secure } \\
& \Rightarrow \text { Easy to use } \\
& \Rightarrow \text { Privacy is maintained }
\end{aligned}
$$

* Parents can securely keep some things away from the reach of the children.

- In the office scenario, files and essentials things are kept completely secured from unauthorized access.

- Smart technology brings ease of use as the drawer can be opened even if hands are occupied elsewhere.

\section{Proposed Algorithm}

1. Open camera and take image then Crop and Convert image to grayscale.

2. Blur image to capture threshold of the image then Call threshold and pass blurred image

3. Call function to find contours and pass a copy of variables and find length of contours.

4. Compute the defects.(lower portion between two fingers).

5. Check total number of defects.

5.1. If number of defects is one then go to next.

5.2. If number of defects is four then close the drawer.

5.3. If gesture not found.

5.3.1. goto step 1.

6. check security mode.

6.1. If security is enabled

6.1.1. The face image is searched in database.

6.1.2. If face matched 
6.1.2.1. Open drawer.

6.1.3. If face not matched,

6.1.4. Exit.

Flow Chart of our proposed work 


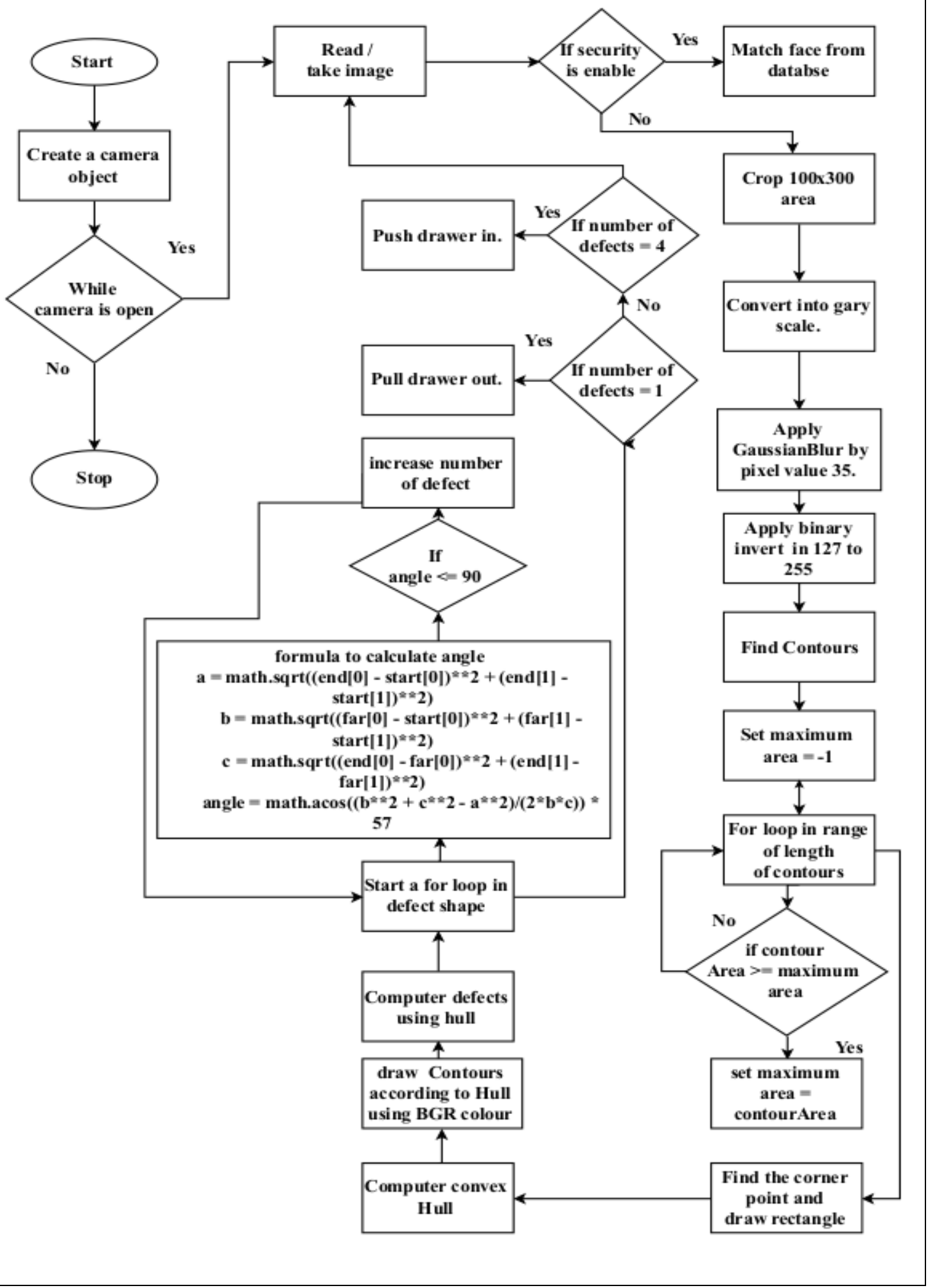

\section{Experiment and Result}

In this section we have shown how the technology is working using gesture sensing. We have set the values upon which the gesture sensing works. If it recognize two fingers like victory symbol the drawer will open upon 
recognition the face if security is enabled. The drawer will be closed if it recognize five fingers of our hand. The following image will show the working strategy of our proposed work.

\section{For opening the drawer}

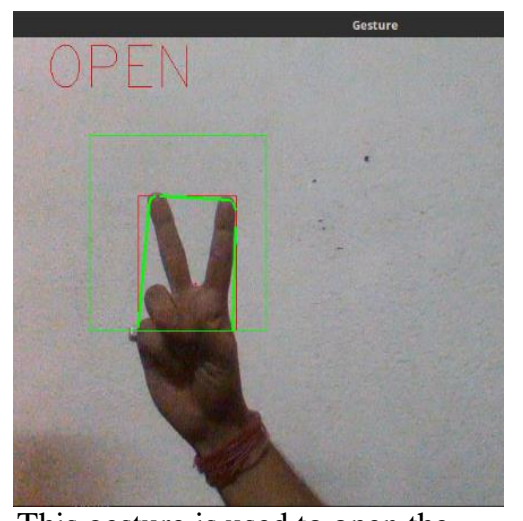

This gesture is used to open the drawer

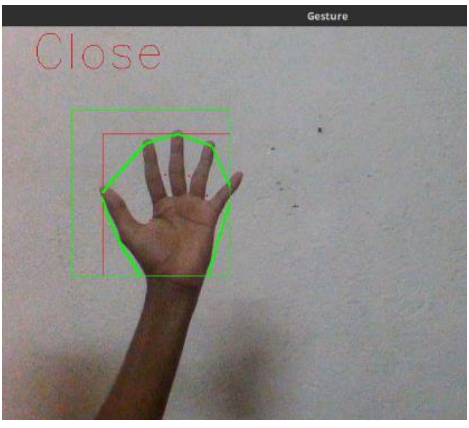

This gesture is used to close the drawer.

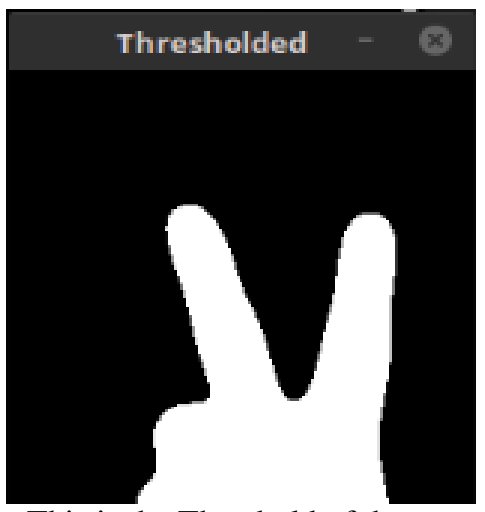

This is the Threshold of the opening gesture.

\section{For closing the Drawer}

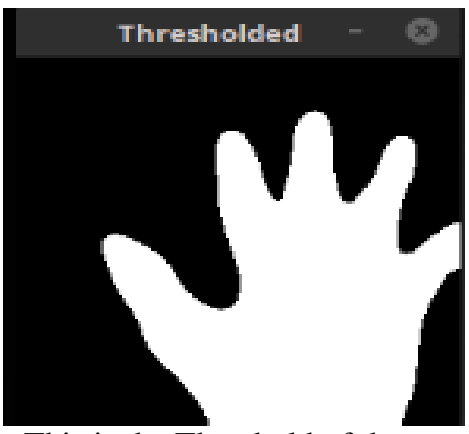

This is the Threshold of the closing gesture

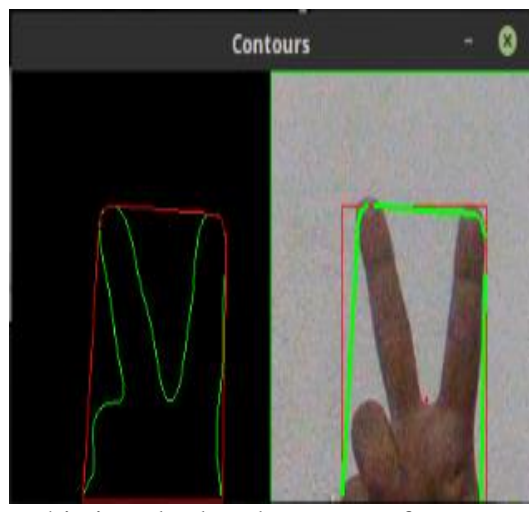

This is calculated contours from the gesture.

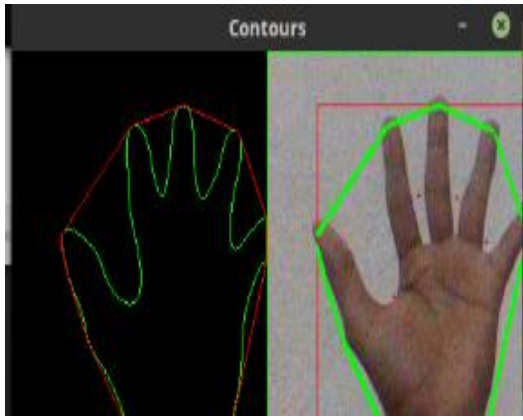

This is calculated contours from the gesture.

\section{V.CONCLUSION}

Initially gesture recognition was with difficult technology like sensor, glove etc. now it becomes easier with webcam, image processing software and gaming tools. In the early research gesture control or recognition process was complex, but now in the era of smarter world it is simple vision technique using hand, head or even whole body gesture. Computer application operating was the main target in the early stage. But now it is widely accepted for ambient device and ubiquitous computing. In recent researches, the more focus have been given to control home appliances, to use mobile device, large screen, table top screen and to manage group work, or even home residents activities. In our research work we have introduced a smart drawer where both simple mode and secure mode is available, i.e. both the purpose of user has been solved. Another most important aspect is now it's really affordable, while it was expensive before. Now a drawer will be easy and also beneficial for use to general people. The simplicity and smartness makes the drawer more acceptable in the society. 


\section{REFERENCES}

[1]. C. A. Pickering, K. J. Burnham and M. J. Richardson, "A Research Study of Hand Gesture Recognition Technologies and Applications for Human Vehicle Interaction," 2007 3rd Institution of Engineering and Technology Conference on Automotive Electronics, Warwick, UK, 2007, pp. 1-15.

[2]. Johnny Accot and Shumin Zhai "Beyond Fitts' Law: Models for Trajectory-Based HCI Tasks" in CHI '97 Proceedings of the ACM SIGCHI Conference on Human factors in computing systems March 22 - 27, 1997 Pages 295-302

[3]. Michael Moyle and Andy Cockburn "Analysing Mouse and Pen Gestures" in the proceeding CHINZ '02 Proceedings of the SIGCHI-NZ Symposium on Computer-Human Interaction Pages 19-24 July 11 - 12, 2002

[4]. Mammen, James, Subhasis Chaudhuri, and Tushar Agarwal. "A two stage scheme for dynamic hand gesture recognition." In Proceedings of the National Conference on Communication, pp. 35-39. 2002.

[5]. Yang, Jie, and Yangsheng Xu. "Hidden markov model for gesture recognition" No. CMU-RI-TR-94-10. CARNEGIE-MELLON UNIV PITTSBURGH PA ROBOTICS INST, 1994.

[6]. Morimoto, Carlos, Yaser Yacoob, and Larry Davis. "Recognition of head gestures using hidden Markov models." Proceedings of the 13th International Conference on Pattern Recognition, 1996 Vol. 3. IEEE, 1996.

[7]. Hong, Pengyu, Matthew Turk, and Thomas S. Huang. "Constructing finite state machines for fast gesture recognition." in the Proceedings Pattern Recognition volume 3, 15th IEEE International Conference 2000

[8]. Sandberg, Anders. "Gesture recognition using neural networks". Diss. Master's Thesis TRITA-NA-E9727 Dept. Numerical Analysis and Computing Science, Stockholm University, 1997. 